\title{
So spiegelt sich der Diabetes im Auge
}

\author{
Das Auge erlaubt als einziges Organ des Körpers einen nicht-invasiven direkten Blick auf ein \\ kapilläres Gefäßnetz. Der Zustand der retinalen Gefäße repräsentiert die übrigen Gefäßnetze \\ des Körpers. Einige Erkrankungen bzw. deren Folgeschäden lassen sich am Auge erkennen. \\ Dazu gehört insbesondere der Diabetes mellitus.
}

_ In den retinalen Gefäßen des Diabetikers laufen die gleichen Prozesse ab wie in anderen kleinen Gefäßen. Sie werden aufgrund einer Endotheldysfunktion nicht ausreichend perfundiert, und Blutbestandteile treten durch eine erhöhte Permeabilität des Endothels ins Gewebe über. Es kommt zu Gefäßverschlüssen, Mikroaneurysmen und retinalen Blutungen. Liegt eine Endotheldysfunktion mit Ischämie vor, werden Wachstumsfaktoren wie VEGF (vascular endothelial growth factor) und FGF (fibroblast growth factor) ausgeschüttet. Vor allem VEGF stimuliert eine krankhafte Gefäßneubildung, erklärte Prof. Gangolf Sauder, Chefarzt der Charlottenklinik für Augenheilkunde in Stuttgart.

Man unterscheidet die nicht-proliferative und die proliferative diabetische Retinopathie sowie das diabetische Makulaödem. Letzteres kann, muss aber nicht zusammen mit der Retinopathie auftreten.

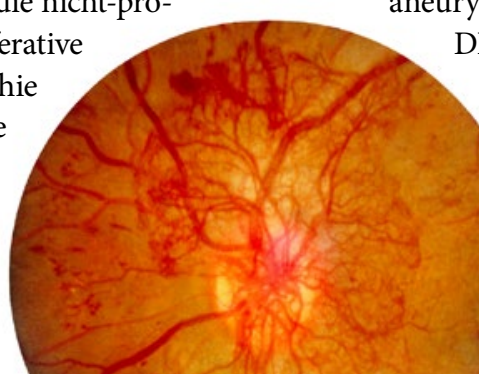

\section{Makulaödem}

Das Makulaödem bemerkt der Patient sofort, sobald es einen Bereich betrifft, der weniger als einen Papillendurchmesser von der Fovea entfernt ist. Denn es beeinträchtigt die Sehkraft. Aber auch wenn das Ödem weiter von der Fovea entfernt ist, hat es klinische Relevanz. Es erfordert aber viel Überzeugungskraft, Patienten, deren Sehvermögen nicht gestört ist, zu vermitteln, dass sie ein schwerwiegendes Problem haben, das zur Erblindung führen kann. Deshalb sollten Diabetiker nicht erst zum Augenarzt kommen, wenn sie schlechter sehen, sondern sich schon vorher regelmäßig kontrollieren lassen sollen.

Breitet sich das Ödem weiter aus auf den gesamten hinteren Pol des Fundus, spricht man von einem diffusen diabetischen Makulaödem. Die Sehkraft des Patienten ist dann massiv beeinträchtigt.

\section{Nicht-proliferative oder proliferative Retinopathie}

Bei einer nicht-proliferativen diabetischen Retinopathie (DRP) finden sich fleck- oder streifenförmige Blutungen und Mikroaneurysmen. Die fortgeschrittene DRP ist charakterisiert durch Mikroaneurysmen und Blutungen in vier Quadranten. Hier kommt meist auch ein Makulaödem hinzu. Neben harten Exsudaten beobachtet man auch Cotton-woolHerde, die Mikroinfarkte in der Nervenfaserschicht repräsentieren.

Bei der proliferativen Diabetische Retinopathie mit zahlreichen Hämorrhagien und Mikroaneurysmen.

diabetischen Retinopathie wuchern neue Gefäße auf der Netzhaut. Oft dringen Gefäße auch in den Glaskörper ein und können dort unter Einfluss von VEGF und FGF fibrovaskuläre Segel bilden, die Zug auf die Netzhaut ausüben und sie ablösen können (traktive Netzhautablösung).

\section{Laser senkt Sauerstoffbedarf}

Die Progression einer leichten bis mittelschweren nicht-proliferativen Netzhauterkrankung kann gebremst werden durch Senkung von Blutzucker und Blutdruck sowie Reduktion des Körpergewichts und Verzicht auf Rauchen.

Eine fortgeschrittene nicht-proliferative Retinopathie ist durch ein optimiertes Diabetes-Management kaum mehr umkehrbar. Sie wird vorzugsweise mit panretinaler Laserkoagulation behandelt. Dabei werden Laserherde von 100-500 $\mu \mathrm{m}$ Größe gesetzt - unter Aussparung des zentralen Makula- und Fovea-Bereichs. Damit wird die Fläche, die mit Sauerstoff versorgt werden muss, reduziert. Dies führt dazu, dass weniger VEGF ausgeschüttet wird. Der Verlauf, der zur Erblindung führen kann, kann hierdurch verzögert werden.

Auch die proliferative diabetische Retinopathie wird mit Laserkoagulation behandelt, wodurch sich die Neovaskularisationen zurückbilden. Das Lasern kann man mit einer intravitrealen Injektion von VEGF-Antikörpern (Bevacizumab, Ranibizumab) kombinieren. Diese hemmen ebenfalls die Neovaskularisation und dichten die Gefäße ab.

\section{Fortschritt Anti-VEGF-Therapie}

Das diffuse Makulaödem ist die Domäne der Anti-VEGF-Therapie. Die Patienten erhalten in Abständen von einem Monat drei intravitreale Injektionen. Man spürt dabei nicht mehr, als wenn man bei geschlossenem Lid mit dem Finger auf das Auge drückt.

\section{Dr. med. Angelika Bischoff}

- 52. Ärztekongress der Bezirksärztekammer Nordwürttemberg, Stuttgart 2017 